\title{
Evaluación de la corrosión en estructuras de contención nuclear mediante el uso de técnicas electroquímicas no destructivas
}

\author{
Corrosion evaluation in nuclear contention structures \\ using electrochemical nondestructive techniques
}

$\underline{\text { I. Martínez }}{ }^{(*)}$, A. Castillo(*), C. Andrade ${ }^{(*)}$

RESUMEN

Son muchos los planteamientos que se realizan en relación al estado de estas estructuras nucleares y a la posibilidad de prorrogar su vida útil, siendo la corrosión uno de los problemas que más preocupa en la actualidad. Por ello, la evaluación y control de la corrosión en estructuras como los liners de los edificios de contención es extremadamente importante para que éste no sea el motivo que limite la vida de estas estructuras.

En el presente trabajo se muestran resultados de la aplicación de técnicas electroquímicas no destructivas en estructuras reales de contención nuclear para la evaluación de la corrosión de armaduras o elementos metálicos embebidos en el hormigón, como son los liners antes mencionados.

$628-9$

Palabras clave: Corrosión; centrales nucleares; liner; técnicas no destructivas.

\section{SUMMARY}

There are many approaches that are made regarding the status of these nuclear structures and the possibility of extending its service life, being corrosion one of the problems that most concern today. Therefore, the corrosion control and evaluation in structures such as the liners of the contention buildings is extremely important to assure that this is not the reason that limits the service life of these structures.

In the present paper application results of non-destructive electrochemical tests for the corrosion evaluation of metallic elements embedded in the concrete of real nuclear contention structures such as the liners mentioned before are presented.
Keywords: Corrosion; nuclear power plants; liner; nondestructive techniques.

\footnotetext{
(*) Centro de Investigación en Seguridad y Durabilidad Estructural y Materiales (CISDEM), CSIC. Persona de contacto/Corresponding author: isabelms@ietcc.csic.es (I. Martínez)
} 


\section{INTRODUCCIÓN}

La durabilidad de las estructuras en Centrales Nucleares es un tema de gran importancia a nivel técnico y con un elevado impacto social en la actualidad. Son muchos los planteamientos que se realizan en relación al estado de estas estructuras nuclerares y a la posibilidad de prorrogar su vida útil. La corrosión de los diferentes elementos metálicos presentes tanto en estructuras aéreas como embebidos en el hormigón es uno de los problemas que más preocupa en la actualidad, y en muchos casos es el factor controlante que podría llegar a limitar la vida útil de estas estructuras.

Por ello, y al igual que sucede en otras tipologías estructurales, es necesario llevar a cabo una tarea de inspección y evaluación de dichas estructuras para tener conocimiento en todo momento de su estado actual y poder predecir, en base a datos objetivos, su evolución futura. Dada la importancia y el nivel de seguridad requerido en estos casos, se plantea la necesidad de diseñar o adaptar métodos que permitan realizar la evaluación de la corrosión sin necesidad de recurrir a ensayos destructivos.

En el caso de estructuras metálicas aéreas o enterradas, el uso de técnicas ultrasónicas o electromagnéticas se ha extendido en los últimos tiempos para detectar fisuras y cuantificar espesores en tuberías y otros componentes (1), pero uno de los mayores problemas se plantea cuando se trata de elementos embebidos en hormigón. En estos casos estas técnicas no proporcionan hasta el momento resultados fiables y es necesario recurrir a otros métodos capaces de proporcionar resultados cuantitativos en relación a la corrosión.

Uno de los casos más críticos y de más difícil estudio son las estructuras denominadas como liner (2), o chapa metálica que delimita la estructura completa del edificio de contención de la Central Nuclear. Esta lámina metálica se encuentra embebida en el contenedor de hormigón armado que constituye la zona de contención, siendo por tanto responsable de proporcionar estanqueidad a dicha zona, y evitando así cualquier tipo de fuga. El liner debe mantener su integridad estructural bajo cualquier condición ambiental o de carga que pueda ocurrir durante toda la vida en servicio de la Central Nuclear, de ahí que los requerimientos para esta estructura sean especialmente estrictos.

Se han encontrado en la bibliografía diferentes publicaciones que estudian un posible fallo del liner en distintas condiciones de carga (3) (4) (5) (6), incluyendo presiones internas, expansiones térmicas, seísmos, retracción o fluencia del hormigón, acción del viento...etc, pero en ningún caso se centran en la detección de un posible problema de corrosión que pueda afectar a su integridad y por tanto a su estanqueidad.

Cambios en el grado de humectación y en el $\mathrm{pH}$ de la disolución presente en los poros del hormigón que protege al liner pueden causar alteraciones en éste capaces de desencadenar un problema de corrosión (7). La difícil accesibilidad del mismo debido a su ubicación (embebido en la cara inferior de una losa de hormigón en ocasiones de casi 1 $\mathrm{m}$ de grosor) así como la necesidad de confirmar su estado de manera no destructiva son factores importantes que llevan a buscar alternativas eficaces para su evaluación.

En el presente trabajo se muestran resultados de la aplicación de técnicas electroquímicas no destructivas en estructuras reales de contención nuclear para la evaluación de la corrosión de armaduras o elementos metálicos embebidos en el hormigón, como son los liners antes mencionados. Estas técnicas se aplican de forma habitual para la detección de la corrosión en estructuras de hormigón armado, tanto en edificación como en obra civil y también han sido utilizadas para la evaluación de otras estructuras nucleares (8).

\section{PARTE EXPERIMENTAL}

\section{- Estructuras evaluadas}

Los edificios de contención evaluados constan de forma general de una estructura laminar de hormigón armado, en ocasiones post-tensado, resistente a altas presiones. La estructura de hormigón armado, que generalmente consiste en muros cilíndricos con una cúpula hemisférica o elipsoidal y como base una losa plana, proporciona el soporte estructural y la resistencia necesaria para soportar las fuerzas debidas a presión inducida (Figura 1). La estanqueidad del edificio hacia el exterior es proporcionada por la lámina continua de acero (liner) relativamente delgada (unos $6 \mathrm{~mm}$ de espesor) que envuelve toda la estructura, desde su losa inferior hasta la cúpula y paredes cilíndricas. Esta lámina está anclada a la lámina de hormigón por perfiles estructurales de acero u otros conectores.

En el presente trabajo se ha evaluado la zona del liner que queda bajo la losa del edificio de contención de dos Centrales Nucleares diferentes. Esta parte está más expuesta a posibles "contaminaciones" 
debido a filtraciones procedentes de diferentes conductos que se encuentran cerca de la losa de refuerzo (Figura 2).

\section{- Metodología de actuación}

Las medidas electroquímicas se realizan directamente sobre la cara de la losa de hormigón accesible desde el propio edificio (opuesta a la cara en la que se encuentra embebido el liner). Se trata de una losa de hormigón armado de espesor variable dependiendo de las necesidades estructurales $(50-80 \mathrm{~cm})$, en la cual se encuentran embebidas dos capas de armado bidireccional con barras de $20 \mathrm{~mm}$ de diámetro distanciadas 0,3 m en ambas direcciones (Figura 2). Tras la segunda capa de armado se encuentra, en contacto directo con el hormigón, el liner o chapa metálica a evaluar, de $6 \mathrm{~mm}$ de espesor. Se ha comprobado que existe continuidad eléctrica entre el liner y las dos capas de armado embebidas en la losa.

En todas las juntas situadas entre la losa y los muros perimetrales existe una capa de material compresible de unos $10 \mathrm{~mm}$ de espesor para asegurar la seguridad de la estructura en caso de seísmo.

Previo a la realización de las medidas electroquímicas se utilizó un detector de armaduras (pachómetro) que permite ubicar mediante corrientes electromagnéticas la primera capa de armado y evitar así lo más posible su interferencia en la medida. No resulta sencilla la detección de las barras de la segunda capa de armado, ya que se encuentran muy cerca del liner. Por ello, debe tenerse en cuenta para la interpretación de resultados que ambos materiales se evaluarán de forma conjunta, ya que es complicado llegar a discernir en las respuestas electroquímicas la parte que corresponde a cada uno de ellos.

Dentro de la superficie de las losas de los edificios de contención descritos, y según se muestra en la Figura 3, se evaluaron un total de 14 zonas de medida: 8 en el edificio de contención denominado como I y otras 6 en el edificio de contención II. En estas zonas se retiró la pintura protectora que recubre la superficie de hormigón en una extensión de 30×30 cm para facilitar la realización de las medidas electroquímicas.

Es importante la verificación de la conexión eléctrica entre el acero de la estructura y el equipo, así como un buen contacto electrolítico entre los electrodos de medida y el recubrimiento de hormigón sobre el cual se realiza la medida.

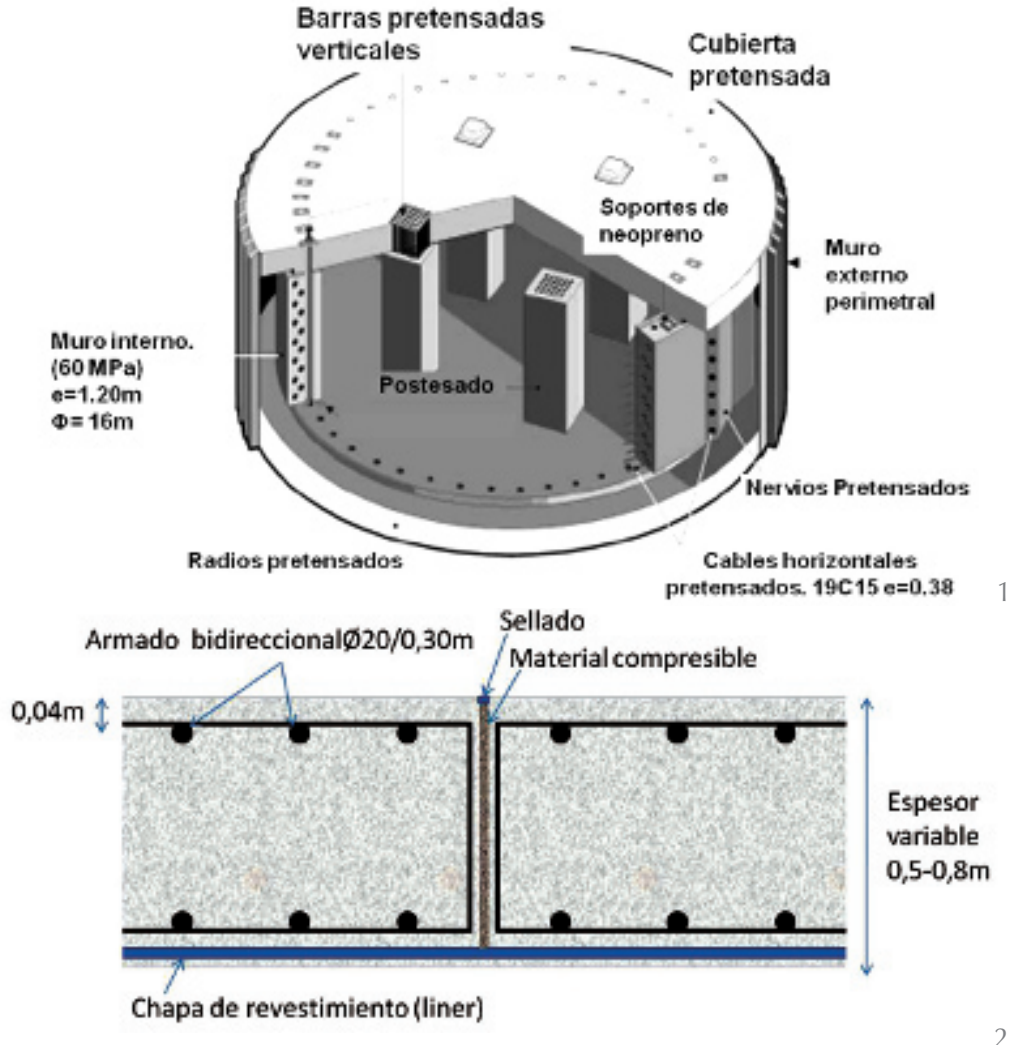

- Técnicas de medida

Las técnicas empleadas para la evaluación del estado de la corrosión de los liners han sido, en todos los casos, técnicas no destructivas que determinan parámetros electroquímicos que informan de forma cuantitativa o cualitativa sobre el estado de corrosión de las armaduras embebidas en el hormigón (9).

Los parámetros electroquímicos registrados han sido:

- Potencial de corrosión $\left(\mathrm{E}_{\text {corr }}\right)$ : Utilizando electrodos de referencia de $\mathrm{Cu} / \mathrm{CuSO}_{4}$

- Resistividad del hormigón $(\rho)$ : Método del pulso galvanostático.

- Intensidad de corrosión $\left(\mathrm{I}_{\text {corr }}\right)$ : Medidante la técnica de la Resistencia de polarización y aplicando el método de confinamiento modulado de la corriente.

\subsection{Medida del potencial de corrosión:}

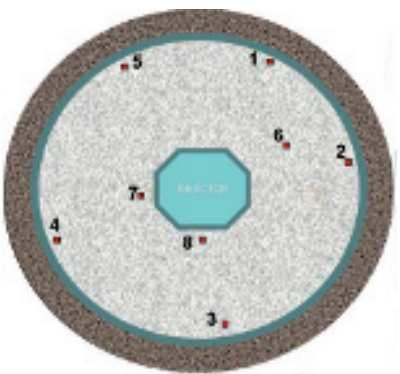

EDIFICIO DE CONTENCIÓN I

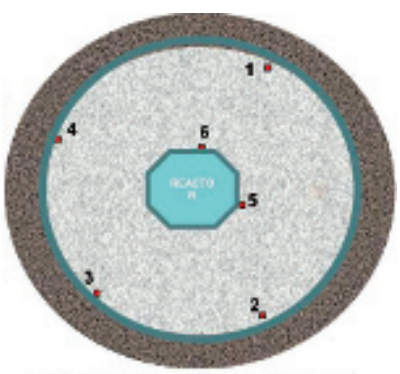

EDIFICIO DE CONTENCIÓN ॥

Proporciona información cualitativa sobre el riesgo de corrosión, pudiéndose establecer unos límites para los cuales la probabilidad de corrosión es elevada, pero sin que se pueda establecer de una forma general y para cualquier condición de exposición una relación constante entre $\mathrm{E}_{\text {corr }}$ y la cinética del proceso de corrosión.

La medida del potencial de corrosión consiste en determinar la diferencia de potencial eléctrico entre el acero del liner conectado a
1. Esquema de la estructura interna del edificio de contención.

2. Sección esquemática de la losa del edificio en la que se realizaron las medidas electroquímicas.

3. Zonas seleccionadas en ambos edificios de contención para su evaluación mediante técnicas electroquímicas. 
4. Medida de I con el Corrosímetro portátil Gecor ${ }^{8}$

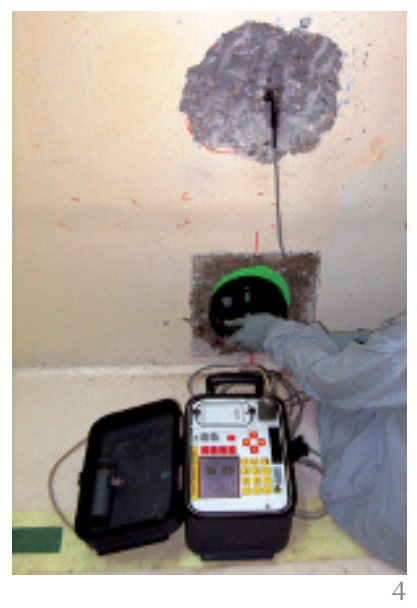

Método patentado por investigadocorrosímetro portátil Gecor ${ }^{08}$. res del CSIC e implementado en el

las armaduras y un electrodo de referencia que se coloca en contacto con la superficie del hormigón. La interpretación de las medidas de potencial de corrosión se suele hacer calificando el riesgo según el criterio dado en la Norma UNE 112083 (10).

\subsection{Medida de la resistividad del hormigón}

Ofrece una visión del grado de saturación del hormigón (11). El grado de saturación afecta directamente a la probabilidad de ausencia o presencia de corrosión, ya que se precisa de una cierta cantidad de agua líquida en los poros del hormigón para que pueda producirse la reacción catódica. La medida de la resistividad del hormigón ayuda por tanto a interpretar el valor de la velocidad de corrosión, I ${ }_{\text {corr }}$ (12) (13), y se han establecido distintos rangos gracias a la experiencia en laboratorio (14).

En el presente trabajo la medida de resistividad se ha llevado a cabo utilizando el denominado método del disco (15) (16).

\subsection{Medida de la velocidad de corrosión}

La medida de la velocidad de corrosión proporciona la cantidad de metal que se transforma en óxido por unidad de tiempo y de superficie. La cantidad de óxido generada está ligada a la fisuración del hormigón y pérdida de adherencia entre el acero y el hormigón. Por otro lado, la pérdida de espesor del metal afecta directamente a la pérdida de capacidad portante de la estructura. La velocidad de corrosión es por tanto un indicativo de la velocidad a la que decrece la capacidad portante de la estructura debido a la pérdida de sección, la pérdida de ductilidad del acero y la pérdida de adherencia entre acero y hormigón. Además, en el caso del liner que aquí nos atañe, una pérdida de espesor total del mismo en un único punto implicaría una pérdida de estanqueidad inadmisible para esta tipología estructural.

Para la medida de la intensidad o velocidad de corrosión se aplica la técnica de la resistencia de polarización, aplicando la ecuación [1] de Stern y Geary (17) que relaciona ambos parámetros.

$$
\mathrm{I}_{\text {corr }}=\mathrm{B} / \mathrm{A} \cdot \mathrm{R}_{\mathrm{p}}
$$

Tabla 1. Criterios para la interpretación de resultados de $I_{\text {corr }}$

\begin{tabular}{|c|c|c|}
\hline $\begin{array}{c}\text { Intensidad de corrosión } \\
\mathrm{I}_{\text {CORR }}\left(\boldsymbol{\mu} \mathrm{A} / \mathbf{c m}^{2}\right)\end{array}$ & $\begin{array}{c}\text { Velocidad de corrosión } \\
\mathbf{V}_{\text {CORR }}(\mu \mathrm{m} / \text { año })\end{array}$ & Nivel de corrosión \\
\hline$<0,1$ & $<1.16$ & Despreciable \\
\hline $0,1 \mathrm{~A} 0,5$ & $1,16 \mathrm{~A} 5,8$ & Baja \\
\hline $0,5 \mathrm{~A} 1$ & $5,8 \mathrm{~A} 11,6$ & Moderada \\
\hline$>1$ & $>11,6$ & Alta \\
\hline
\end{tabular}

En ella B es la constante de Tafel (se asume un valor de $26 \mathrm{mV}$ para el hormigón armado) (18), y A sería la superficie del acero medida.

El valor de la intensidad de corrosión, $\mathrm{I}_{\text {corr }}$ se suele expresar como una densidad de corriente, en $\mu \mathrm{A} / \mathrm{cm}^{2}$. Estos valores se traducen en pérdida de sección del acero mediante la aplicación de la ley de Faraday, por la cual obtendremos las equivalentes velocidades de corrosión en $\mu \mathrm{m} / \mathrm{año}$.

Para realizar la medida de $\mathrm{I}_{\text {corr }}$ en laboratorio (18), normalmente se utiliza un contraelectrodo de la misma dimensión que el electrodo de trabajo y paralelo al mismo. El primer problema que se presenta para la medida de $\mathrm{I}_{\text {corr }}$ in situ es que no se dispone de un electrodo auxiliar de las mismas dimensiones que la armadura a medir, por lo que normalmente se coloca un contraelectrodo de dimensiones reducidas sobre la superficie del hormigón. Este hecho que dificulta el cálculo de la superficie de acero que está siendo polarizada ya que se genera una distribución no regular de las líneas de campo.

En el presente trabajo, las medidas de $\mathrm{I}_{\text {corr }}$ se han realizado mediante "el método de confinamiento controlado de la corriente" (19) (Figura 4). Es un método galvanostático para la medida de $I_{\text {corr }}$ a partir de la $R_{p}$ en el cual se utiliza un anillo de guarda para el confinamiento de la corriente. Realiza una buena estimación del área afectada por la señal gracias a la presencia de dos sensores de campo eléctrico situados entre ambos contraelectrodos que controlan la corriente que se introduce en cada momento por el anillo de guarda. El método ha sido calibrado mediante la realización de ensayos gravimétricos (20).

Los rangos para la interpretación de resultados de $\mathrm{I}_{\text {corr }}$ se han obtenido a partir de la experimentación, mediante la calibración de la técnica por ensayos gravimétricos. En la Tabla 1 se presentan los criterios a tener en cuenta para la interpretación de resulta$\operatorname{dos}(21)(22)$.

\section{RESULTADOS}

\section{a) Potencial de corrosión ( $\left.E_{\text {corr }}\right)$}

En la Figura 5 se presentan los valores de potencial de corrosión (vs $\mathrm{Cu} / \mathrm{CuSO}_{4}$ ) del metal medidos en las 8 zonas escogidas de la losa dentro del Edificio de Contención I en dos inspecciones realizadas en el intervalo de tiempo de 27 meses. La Figura 6 muestra los mismos valores registrados en el Edificio de contención II. Cada uno de 


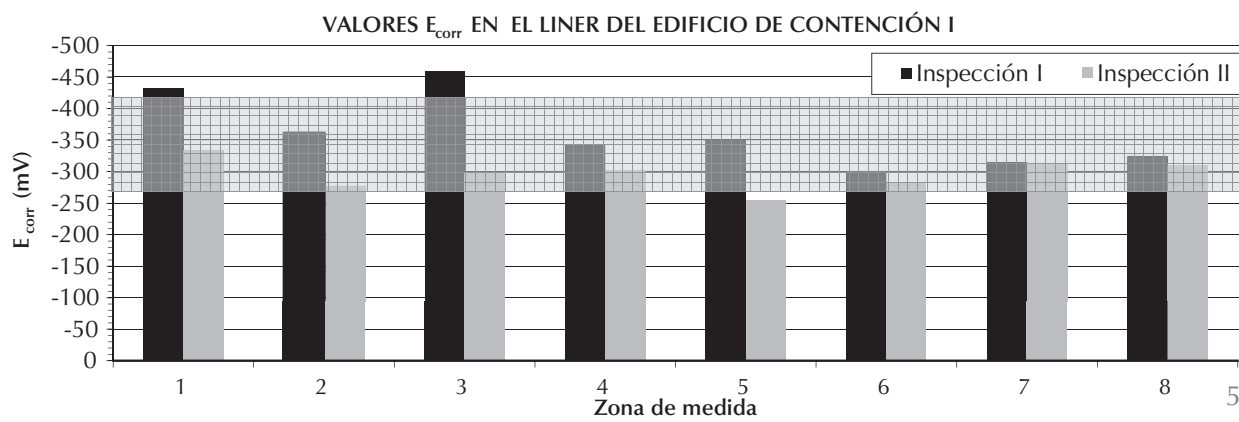

los valores mostrados en la figura representa valor promedio de nueve determinaciones realizadas dentro de cada zona de medida.

En el caso del Edificio de Contención I, en la comparativa de los resultados de ambas inspecciones se observa en general una variación hacia valores más positivos de los potenciales medidos, indicativo de una disminución del riesgo de corrosión en la estructura. Si bien en un primer momento se midieron valores entre -300 y $-470 \mathrm{mV}$ (valor máximo medido en la zona 3), tras la segunda inspección en ningún punto se sobrepasó el valor umbral indicativo de un elevado riesgo de corrosión, situándose todos los valores entre -250 y $-330 \mathrm{mV}$. Estas diferencias en los valores de potencial de corrosión pueden deberse a una variación en la presencia de oxígeno (presión parcial) en la interfase acero-matriz cementicia, siendo ésta menor en la segunda inspección que en la primera. Una mayor concentración de oxígeno va ligada a una menor presencia de humedad, aunque no siempre es así ya que en casos de elevado contenido de humedad (próxima al 100\%) la difusión del oxígeno a través de los poros del hormigón disminuye.

En el edificio de contención II (Figura 6), no se registraron en ningún caso valores de potencial de corrosión que indicaran un alto riesgo de corrosión, situándose todos los valores entre -180 y $-350 m V$.

Debe tenerse en cuenta que en ningún caso este parámetro está cuantificando la corrosión que presenta la estructura, para ello será necesario evaluar la velocidad de corrosión.

\section{b) Resistividad ( $\rho)$}

En la Figura 7 y la Figura 8 se presentan los valores de resistividad del hormigón registrados en los edificios de contención I y II respectivamente. Como en el caso del potencial de corrosión, cada uno de los valores mostrados en la figura representa valor promedio de nueve determinaciones realizadas dentro de cada zona de medida.
En el Edificio de contención I, las resistividades del hormigón de la losa se encontraban en la primera inspección entre 3,6 y $13,5 \mathrm{~K} \Omega \mathrm{cm}$. En la segunda inspección realizada no se observa aumento alguno del valor de la resistividad medida, situándose todos los valores entre 4 y $9 \mathrm{~K} \Omega \mathrm{cm}$. Se puede interpretar por tanto que el contenido de humedad del hormigón ha aumentado
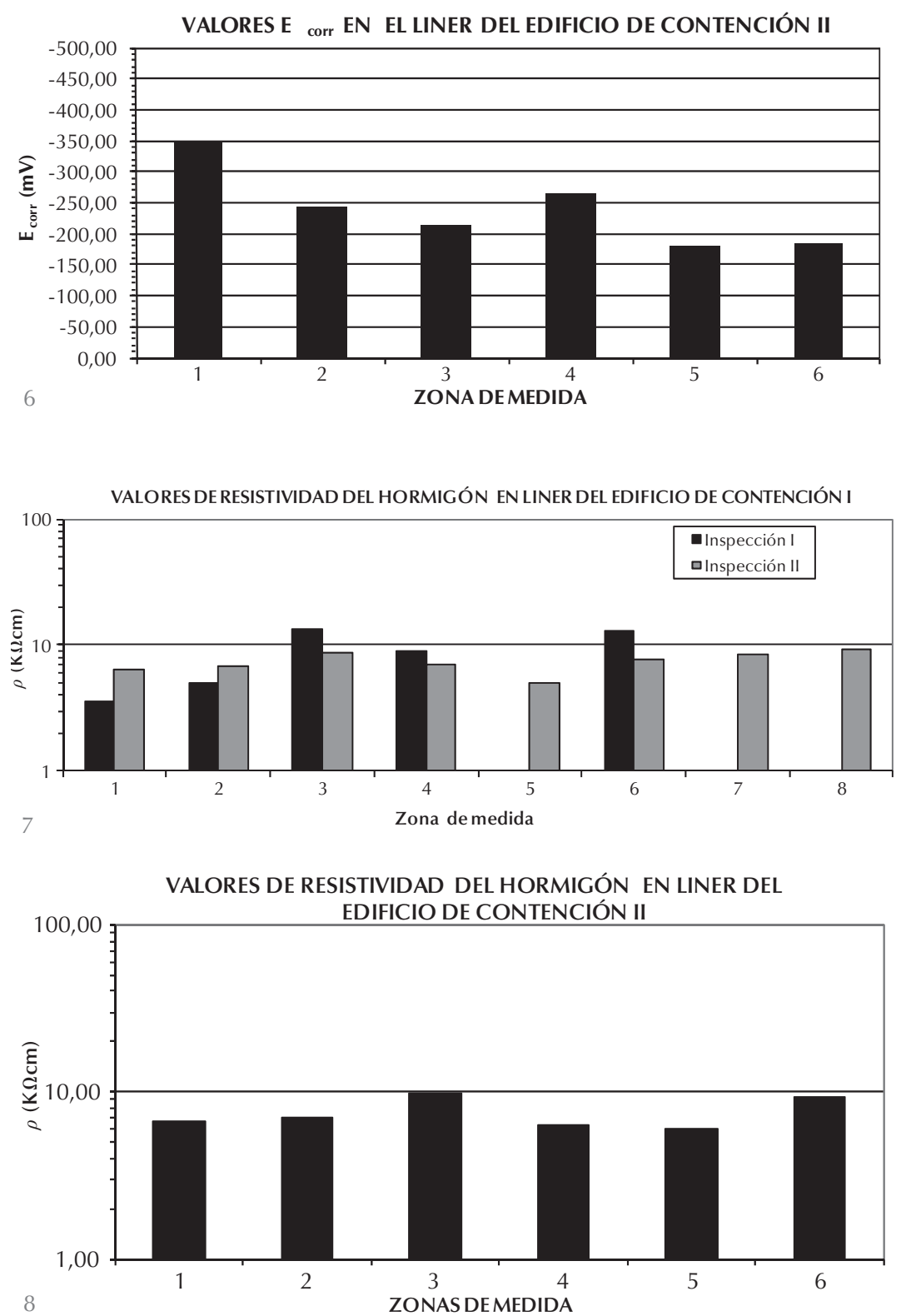
9. Medidas de velocidad de Velocidad de Corrosión realizadas durante las inspecciones en el edificio de contención I.

10. Medidas de velocidad de Velocidad de Corrosión realizadas durante las inspecciones en el edificio de contención II. ligeramente, aunque dada la alta presencia de humedad, esta variación no tendría por qué estar ligada a una mayor presencia de oxígeno. De hecho, los valores de Ecorr previamente comentados indicarían una disminución del mismo.

En el caso del Edificio de Contención II, las resistividades medidas son muy similares a las registradas en el caso anterior, situándose en todos los casos entre 6 y $10 \mathrm{~K} \Omega \mathrm{cm}$. El hecho de encontrar de forma general resistividades del hormigón inferiores a $10 \mathrm{~K} \Omega \mathrm{cm}$ indican un alto grado de humectación en el mismo.

\section{c) Intensidad de corrosión ( $\left.I_{\text {corr }}\right)$}

En la Figura 9 y la Figura 10 se muestran los valores de velocidad de corrosión medidos en el los edificios de contención I y II respectivamente.

Dada la posibilidad, mencionada anteriormente, de que la medida de la velocidad de corrosión se esté viendo influenciada por la presencia del armado, se han realizado estimaciones del valor de $\mathrm{I}_{\text {corr }}$ considerando el área total que incluye la zona correspondiente al liner y a la segunda capa de armado incluidas en la zona de influencia del sensor de medida.

Pese a que en la mayoría de las zonas medidas en el edificio de contención I se ha superado el umbral de despasivación de $0,2 \mu \mathrm{A} / \mathrm{cm}^{2}$, sólo en la zona de media n. ${ }^{\circ} 1$ se ha superado el valor de $0,5 \mu \mathrm{A} / \mathrm{cm}^{2}$, por lo que puede decirse en general que las velocidades de corrosión son bajas. No se encuentran variaciones significativas que puedan indicar un descenso de la corrosión de la primera a la segunda inspección realizadas. En el caso del edificio de contención II solo dos de las zonas evaluadas han superado el umbral de despasivación, presentando en esos casos velocidades de corrosión bajas.

\section{DISCUSIÓN}

A la vista de los resultados obtenidos de los diferentes liners en las inspecciones realizadas, se pueden sacar las siguientes reflexiones, enmarcándolas en las conclusiones extraídas de las medidas de los parámetros electroquímicos:

Fiabilidad en el riesgo de corrosión deducido de la medida de $\mathrm{E}_{\text {corr }}$ :

En la Figura 11 se muestra la representación de la totalidad de los resultados de $\mathrm{I}_{\text {corr }}$ obtenidos en las distintas inspecciones realizadas en relación a los valores de $\mathrm{E}_{\text {corr }}$ registrados en esos mismos puntos en el momento de la medida. Se observa cómo es difícil encontrar una correlación clara entre ambos parámetros. No obstante, siguiendo los criterios antes mencionados para deter-
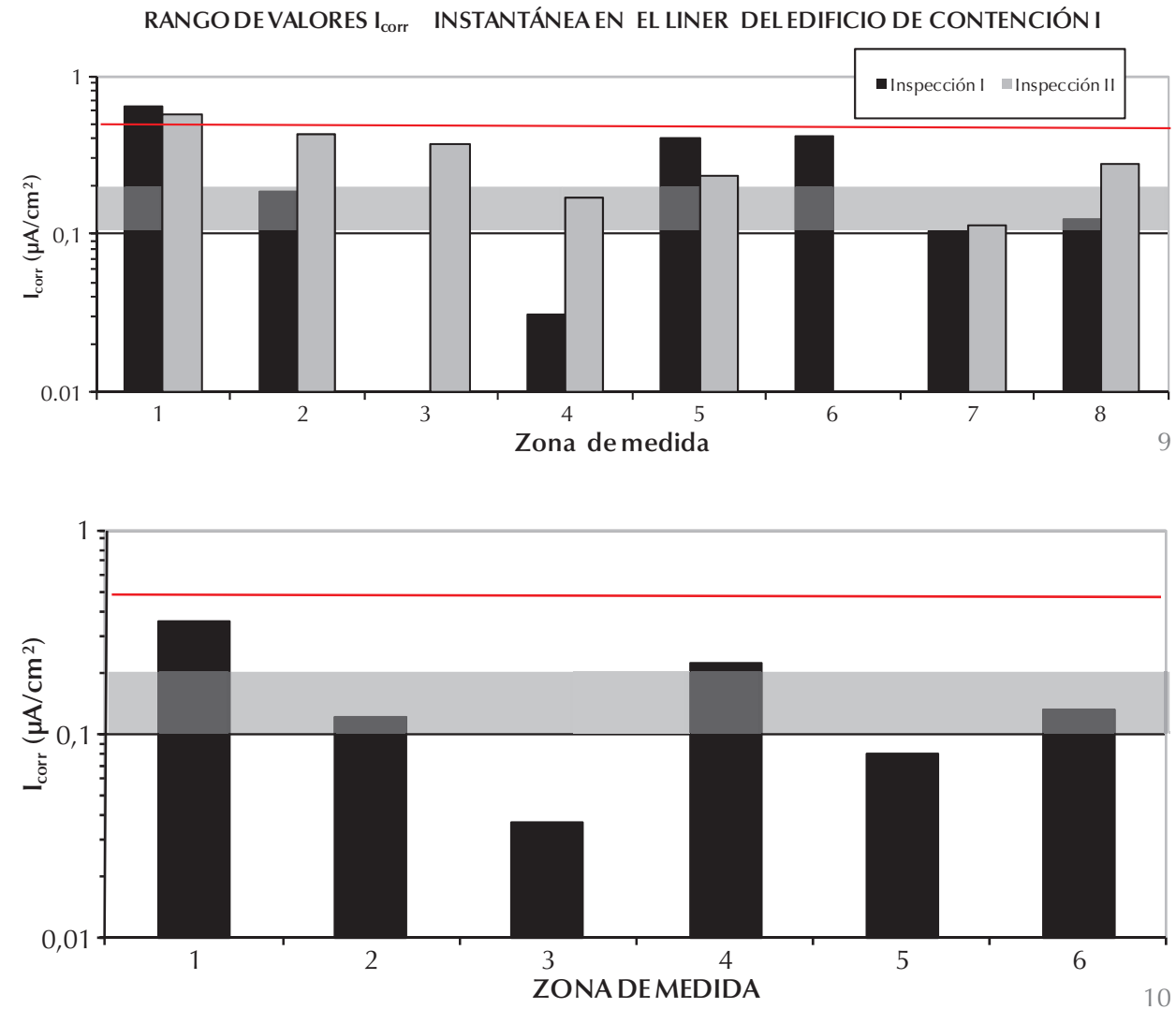
minar el riesgo de corrosión a partir de la medida de $\mathrm{E}_{\text {corr }}$ (10) y las recomendaciones dadas para la interpretación de los valores de $\mathrm{I}_{\text {corr }}$ en cuanto a la cuantificación de la corrosión (22), podemos concluir en base a lo observado en la Figura 11 que, cuando el valor de $\mathrm{E}_{\text {corr }}$ indica un bajo riesgo de corrosión (a la derecha de la zona vertical sombreada), todos los valores de $\mathrm{I}_{\text {corr }}$ medidos se encuentran por debajo de $0,5 \mu \mathrm{A}$ / $\mathrm{cm}^{2}$ (línea discontinua horizontal). Si bien este valor de $0,5 \mu \mathrm{A} / \mathrm{cm}^{2}$ se encuentra por encima del umbral de despasivación (establecido entre 0,1 y $0,2 \mu \mathrm{A} / \mathrm{cm}^{2}$ ), se interpreta como un valor de corrosión bajo, debido a las escasas pérdidas de sección que podría producir el proceso de deterioro en un periodo de tiempo relativamente largo. Para un riesgo de corrosión elevado (valores a la izquierda de la zona vertical sombreada), se observa cómo el único valor registrado, presenta una velocidad de corrosión superior a $0,5 \mu \mathrm{A} / \mathrm{cm}^{2}$, por lo que los criterios establecidos parecen en principio ser coherentes. El problema está en que un elevado porcentaje de las medidas realizadas indican valores de $\mathrm{E}_{\text {corr }}$ dentro de la zona sombreada, en la cual existe una incertidumbre grande en relación a su riesgo de corrosión. Si observamos los valores de I registrados en este rango de valores de $\mathrm{E}_{\text {corr }}$ (zona sombreada) podemos comprobar que, efectivamente, para valores de $\mathrm{E}_{\text {corr }}$ similares los valores de I varr varian desde 0,02 a $0,6 \mu \mathrm{A} / \mathrm{cm}^{2}$.

Por todo ello, se corrobora el hecho de que, si bien la medida del potencial de corrosión nos proporciona valores de manera rápida y sin precisar de un equipamiento complejo, estas medidas no van a dar en muchos casos información fiable en relación al estado de corrosión de la estructura, no siendo el parámetro de $\mathrm{E}_{\text {corr }}$ un indicador de la pasividad o actividad del metal. Es necesario recurrir por tanto a la medida de Icorr para cuantificar la cinética del proceso.

\section{Relación entre la Resistividad ( $\rho)$ y la $I_{\text {cor }}$}

Como se ha indicado anteriormente, existe una correlación entre la velocidad de corrosión que presenta un acero embebido en hormigón y la resistividad (contenido de agua líquida en los poros) de éste hormigón (12) (13). Debemos remarcar que el control de la resistividad en la velocidad de corrosión sólo se produce cuando el metal embebido está sufriendo un proceso de corrosión activo. Si el metal se encuentra en estado pasivo, la resistividad no se relaciona con el valor de la velocidad de corrosión.

Teniendo en cuenta que la resistividad del hormigón de las losas evaluadas en los

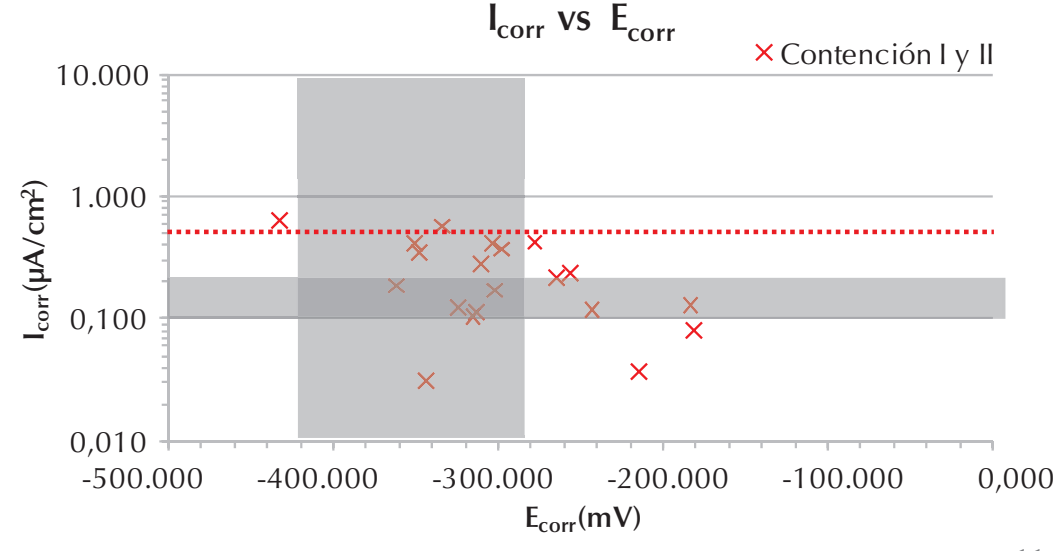

11

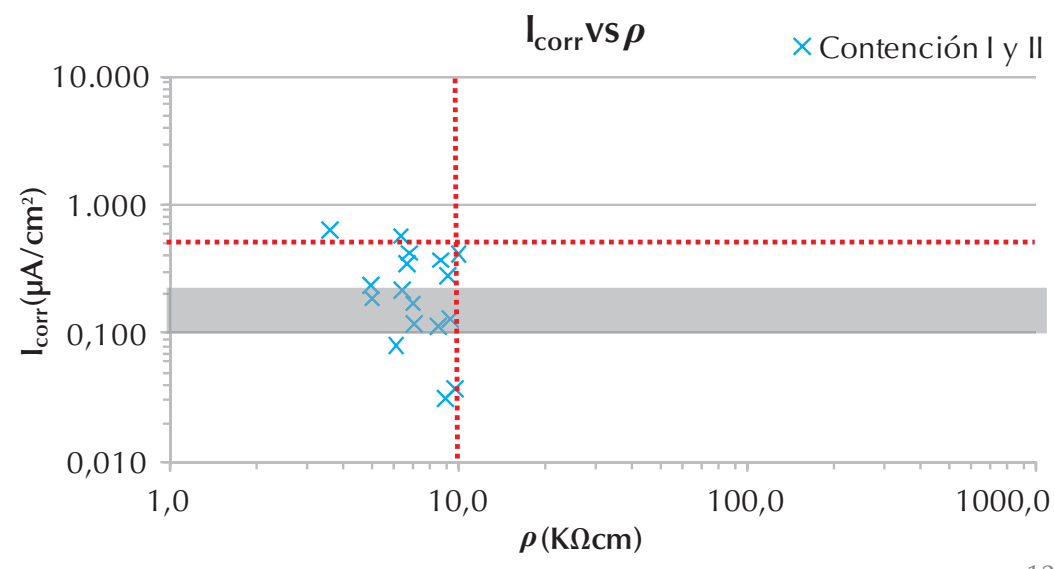

dos edificios de contención se encuentra en todos los casos entre 3 y $13 \mathrm{k} \Omega . \mathrm{cm}$, la posibilidad de tener un cierto riesgo de corrosión es elevado, ya que el hormigón se encuentra con un alto grado de humectación. Para confirmar si esta condición de elevada humedad induce o no corrosión de los aceros, la Figura 12 presenta la correlación entre ambos parámetros. Se observa que la inmensa mayoría de los valores de I se encuentran por encima del umbral de despasivación de $0.1 \mu \mathrm{A} / \mathrm{cm}^{2}$, lo cual sería coherente con las bajas resistividades registradas.

En cualquier caso, el hecho de que los valores de resistividad medidos sean tan extremadamente bajos, aproximadamente un orden de magnitud inferior a la que normalmente se mide en estructuras aéreas de hormigón armado (12), puede ser debido precisamente a la presencia del liner. Debemos tener en cuenta que se trata de una chapa metálica continua embebida en el hormigón de la losa en la cara opuesta a la de medida (Figura 2). El método de medida utilizado para la determinación de la resistividad del hormigón (15) contempla la presencia de barras de acero discretas embebidas en el hormigón, y establece una expresión determinada para el factor de
11. Correlación entre las medidas de $\mathrm{I}_{\text {yor }}$ y $\mathrm{E}_{\text {corr }}$ tomadas en los edificios de contención I y II.

12. Correlación entre las medidas de I y $\rho$ tomadas en los edificios de contención I y II. 
13. Espesores residuales estimados a partir de los valores de I forma a aplicar debido a la geometría de la celda considerada. Al variar esta configuración en este caso particular incluyendo en el sistema la presencia del liner, se varía en cierto modo esta geometría, por lo que la interpretación de estos resultados deberá estudiarse en mayor profundidad.

\section{Interpretación de los resultados de I : Es- timación de la pérdida de espesor de los liners}

Como se ha mencionado en un principio, la medida de la velocidad de corrosión proporciona información directa sobre la pérdida de propiedades resistentes de un elemento estructural y por tanto, sobre la vida útil residual de dicho elemento.

En el caso de utilizar este parámetro como herramienta para la evaluación de la vida útil de los liners de edificios de contención en centrales nucleares, el mayor interés es conocer el espesor de éstos en las zonas más críticas, dado que su finalidad final se centra en asegurar la estanqueidad de la estructura. Por ello, con la línea de investigación iniciada en este campo se pretende realizar una evaluación continua de las estructuras en estudio para poder estimar en cada momento las necesidades de mantenimiento de la estructura así como predecir su comportamiento futuro y su vida útil.

La penetración de ataque acumulada, $\mathrm{P}_{\mathrm{x}^{\prime}}$ puede calcularse a partir de la expresión [2]:

$$
P_{x}=0,0115 \mathfrak{j} I_{\text {corr }}^{R E P} \mathbf{j} t_{p}
$$

siendo I ${ }_{\text {corr }}^{\text {REP }}$ el valor de velocidad de corrosión reperesentativa durante el periodo de tiempo $t_{p^{\prime}}$ en años, que va desde el inicio de la corrosión hasta el momento de la medida, y 0,0115 el factor de conversión de $\mu \mathrm{A} / \mathrm{cm}^{2}$ en mm/año deducido a partir de la ley de Faraday para el acero. Para poder aplicar la anterior expresión es necesario conocer o estimar el momento de inicio de

\section{Espesor residual}

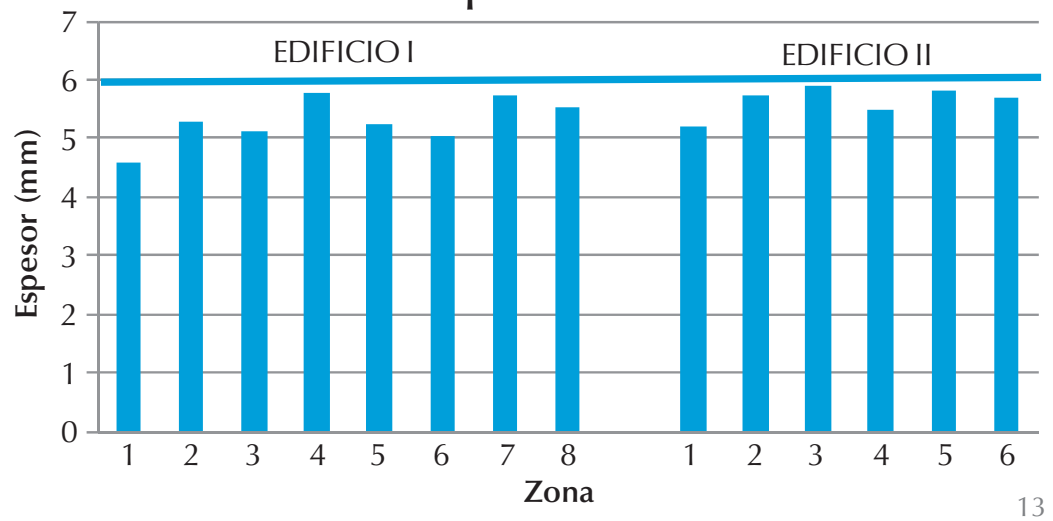

la corrosión para así determinar $\mathrm{t}_{\mathrm{p}}$ de la forma más precisa posible.

Cuando se trata de un proceso de corrosión localizada, la máxima penetración de ataque, $\mathrm{P}_{\text {pit' }}$ puede calcularse a partir de la expresión [3], en la cual se incluye un factor $\alpha$. Normalmente este valor oscila entre $3 y$ 10 (23).

$$
P_{\text {pit }}=0,0115 \cdot I_{\text {corr }}^{R E P} \cdot t_{p} \cdot \alpha=0,115 \cdot I_{\text {corr }}^{R E P} \cdot t_{p}
$$

En el caso que nos ocupa, y dado que no es posible determinar en qué momento se ha producido en cada caso la despasivación del liner (inicio de la corrosión) sin la realización de los correspondientes ensayos destructivos, nos pondremos del lado de la seguridad considerando como $t_{p} 20$ años (tiempo similar a la vida en servicio de las estructuras evaluadas). Consideraremos también la posibilidad de corrosión localizada, con un factor de picadura máximo, $\alpha=10$. En la Figura 13 se presentan los espesores residuales calculados teniendo en cuenta las hipótesis de partida descritas y aplicando la ecuación [3]. Se observa como el mínimo espesor estimado siguiendo estos criterios sería de 4,6mm (zona 1 del edificio de contención). En el resto de zonas evaluadas las pérdidas de espesor estimadas serían inferiores en todos los casos a $1 \mathrm{~mm}$, por lo que se puede concluir que, aunque se haya detectado corrosión en algunas de las zonas de los liners evaluados, incluso en el supuesto de que esta corrosión fuera localizada, en ningún caso se vería reducida la vida en servicio prevista para estas estructuras.

\section{CONCLUSIONES}

El uso de técnicas electroquímicas no destructivas ha resultado satisfactorio para la evaluación de posibles deterioros debidos a procesos corrosivos en estructuras tipo liner de los edificios de contención de centrales nucleares. De los resultados obtenidos en el presente trabajo se puede además concluir lo siguiente:

- El potencial de corrosión aunque en algunos casos permite establecer de manera general el riesgo de corrosión en el que se encuentran las estructuras en estudio, no es capaz de discernir entre estados activos y pasivos del acero, por lo que es necesaria la evaluación de la velocidad de corrosión para cuantificar la cinética del proceso.

- Los bajos valores de resistividad medidos en ambos casos estudiados indican un alto grado de humectación en el hor- 
migón. Esta presencia de agua en los poros del hormigón es capaz de acelerar la corrosión que pudiera producirse en la estructura en el caso de presencia de agentes agresivos en el hormigón que pudieran descender el $\mathrm{pH}$ del mismo.

- Se ha confirmado que en muchos casos se ha producido la despasivación de la armadura, si bien, los niveles de corrosión alcanzados son bajos, causando pérdidas de sección en el liner hasta el momento poco significativas.
Aunque se haya detectado corrosión en algunas de las zonas de los liners evaluados, incluso en el supuesto de que esta corrosión fuera localizada, en ningún caso se vería reducida la vida en servicio prevista para estas estructuras.

\section{AGRADECIMIENTOS}

Los autores del presente trabajo agradecen la ayuda prestada por parte de la propiedad de las Centrales Nucleares para la realización de los trabajos de evaluación.

\section{BIBLIOGRAFÍA}

(1) Komura, I; Hirasawa, T; Nagai, S; Takabayashi, J; Naruse, K.: "Crack detection and sizing technique by ultrasonic and electromagnetic methods." Nuclear Engineering And Design, Vol. $206 \mathrm{n}^{\circ}$ 2-3 (2001), pp. 351-362. presented at 25th MPA Seminar in Stuttgart, Germany, OCT 07-08, 1999.

(2) Doyle, J.M.; Chu, S.L.: "Some structural considerations in the design of nuclear containment liners" Nuclear and engineering design, Vol. 16 n 3 (1971), pp. 294-300.

(3) D.J. Naus; B.R. Ellingwood; H.L. Graves III.: "Methods for assessing NPP containment pressure boundary integrity", Nuclear engineering and design, Vol. 228 n 1-3 (2004), pp.55-72.

(4) Olowokere, O.: "Failure of stiffened steel liners for nuclear reactor containments structures under construction", Computers and structures, Vol. 19 n4 (1984), pp 669-672.

(5) Granger, L; Rieg, C.Y. et al.: "Containment evaluation under severe accidents (CESA): synthesis of the predictive calculations and analysis of the first experimental results obtained on the Civaux mock-up", Nuclear engineering and design, Vol. 209 n 1-3 (2001), pp. 155-163.

(6) Basha, S.M.; Singh, R.K. et al.: "Predictions of ultimate load capacity for pre-stressed concrete containment vessel model with BARC finite element code ULCA", Annals of Nuclear Energy, Vol. 30 n 4 (2003), pp. 437-471.

(7) Pourbaix, M.; "Atlas of electrochemical equilibria in aqueous solutions". NACE, Cebelcor, 1976.

(8) Andrade, C.; Martínez, I; Castellote, M; Zuloaga, P.: "Some principles of service life calculation of reinforcements and in situ corrosion monitoring by sensors in the radioactive waste containers of El Cabril disposal (Spain)". Journal of Nuclear Materials Vol. 358 n $^{\circ}$ 2-3 (2006), pp. 82-95.

(9) Andrade, C; Martínez, I; Alonso, C; Fullea, J.: "New advanced electrochemical techniques for on site measurements of reinforcement corrosion". Materiales de construcción, Vol. 51 n²63-264 (2001), pp. 263-264.

(10) UNE 122083, "Medición del potencial de corrosión libre en estructuras de hormigón armado."

(11) Millard, S.G; Gowers, K.R.: "Resistivity assessment of in-situ concrete: the influence of conductive and resistive surface layers", Proc. Inst. Civil Engrs. Struct. \& Bldgs, Vol. 94 n 4 (1992), paper 9876, pp.389-396. Doi: 10.1680/istbu.1992.21502

(12) Alonso, C; Andrade, C; Gonzalez, J.A.: "Relation between concrete resistivity and corrosion rate of the reinforcements in carbonated mortar made with several cement types". Cement and concrete research, Vol. 118 n 5 (1988), pp 687-698.

(13) Feliú, S; Gonzalez, J.A; Feliú, S. Jr; Andrade, C.: "Relationship between conductivity of concrete and corrosion of reinforcing bars". British Corrosion Journal Vol. $24 \mathrm{n}^{\circ} 3$ (1989).

(14) RILEM Recommendations of TC 154-EMC: Electrochemical techniques for measuring metallic corrosion. "Test methods for on site measurement of resistivity of concrete". Materials and structures, Vol. 33 (2000).

(15) Feliú, S; Andrade, C; González, J.A; Alonso, C.: "A new method for in-situ measurement of electrical resistivity of reinforced concrete". Materials and structures, Vol. $29 \mathrm{n}^{\circ}$ 6 (1996), pp 362-365. Doi: 10.1007/BF02486344

(16) Newman, J.: "Resistance for flow of current to a disk", Journal of the electrochemical society, Vol. 113 n 5 (1966), pp 501-502.

(17) Stern M, Geary A. J. "A Theoretical analysis of the slope of the polarization curves". J. Electrochem. Soc, Vol. 104 n $^{\circ} 1$ (1957), pp 56-63. 
(18) Andrade, C; González, J.A.: "Quantitative measurement of corrosion rate of reinforcing steels embedded in concrete using polarization resistance measurements". Werkstoffe und korrosion, Vol. 29 (1978) p 515.

(19) Feliú, S; González, J.A; Feliú, S. Jr; Andrade, C.: "Confinement of electrical signal for in-situ measurements of polarisation resistance in reinforcement concrete". Mater. J. ACI (1990) pp. 457-460.

(20) Andrade, C; Martínez, I.: "Calibration by gravimetrics losses of electrochemical corrosion rate measurement using modulated confinement of the current". Materials and Structures, Vol. 38 n 9 (2005), pp. 833-841.

(21) Andrade, C; Alonso, C; González, J.A.: "An initial effort to use the corrosion rate measurement for estimating rebar durability. Corrosion rates of steel in concrete", ASTM STP 1065, Berke, N.S; Chaker, V; Whiting, Eds; American Society for testing and materials. pp 29-37 Philadelphia, 1990.

(22) Andrade, C; Alonso, C; Gulikers, J; Polder, R; Cigna, R; Vennesland, O; Salta, M.: Test methods for on-site reinforcement corrosion rate measurement of steel reinforcement in concrete by menas of the Polarization Resistance method. RILEM Recommendation of TC-154 "Electrochemical Techniques for measuring metallic corrosion". Materials and Structures, Vol. 37 (2004), pp 623.643.

(23) González, J.A; Andrade, C; Alonso, C; Feliú, S.: "Comparison of rates of general corrosion and maximum pitting penetration of concrete embedded steel reinforcement". Cement and Concrete Research, Vol. 25 nº 2 (1995), pp. 257-264. 